Knie J. 2019 1 1:3-4

https://doi.org/10.1007/s43205-019-00021-3

๑) Springer Medizin Verlag GmbH 2019

F. Kaufmann - P. Herrmann - A. Sittig - M. Kretz

Springer Medizin Verlag GmbH, Heidelberg, Deutschland

\title{
Knie Journal - Willkommen bei Springer Medizin
}

Sehr geehrte Leserinnen und Leser, sehr geehrte Vertreter und Mitglieder der Deutschen Kniegesellschaft,

wir freuen uns sehr, dass die Deutsche Kniegesellschaft die neue Zeitschrift Knie Journal gemeinsam mit dem Springer Medizin Verlag gegründet hat, und bedanken uns für das in uns gesetzte Vertrauen.

Vor ungefähr einem Jahr fanden erste Gespräche zur Konzeption einer neuen Zeitschrift statt, nun halten Sie das Ergebnis eines Jahres intensiver Zusammenarbeit von Herausgebern und Verlag in den Händen.

Zentrales Anliegen war es, ein ausschließlich auf die Erkrankungen und Verletzungen des Kniegelenks und des periartikulären Bewegungsapparats ausgerichtetes deutschsprachiges Journal zu entwickeln, das die Informationsbedürfnisse aller auf diesem Gebiet tätigen Disziplinen gezielt erfasst. Das Knie Journal trägt damit der hohen Spezialisierung Rechnung, die im Fachgebiet Orthopädie \& Unfallchirurgie seit vielen Jahren zu beobachten ist.

Die neue Zeitschrift möchte verlässliche Quelle in der wachsenden Informationsflut sein, indem wichtige aktuelle Publikationen und Forschungsergebnisse gesichtet, gewichtet und für den Leser bezüglich der klinischen Relevanz bewertet werden. Die sorgfältige Begutachtung der Beiträge ist dabei guter Standard.

Und: Alle Beiträge sind in deutscher Sprache abgefasst, dies ist bei der täglichen Fortbildung ein stets geäußertes Bedürfnis unserer Leser.

\section{Vielfältige Rubriken und Formate}

- Jede Ausgabe mit praxisnahen Übersichten zu einem interdisziplinären Schwerpunktthema

- „CME Zertifizierte Fortbildung“ mit der Möglichkeit, bequem Fortbildungspunkte zu sammeln

- Kompakte Information und konkrete Handlungsanweisung in den Rubriken Operative Techniken, Handlungsalgorithmus und Kasuistiken

- Nachrichten und Informationen aus der Fachgesellschaft

\section{Komfortable Online-Nutzung}

- Beiträge sind dauerhaft auf SpringerMedizin.de und SpringerLink verfügbar

- Responsives Design für gängige Devices und Bildschirmgrößen (Smartphone, Tablet, Notebook/PC)

- Beiträge als PDF, HTML-Version und ePaper

- Online-first-Publikation der Beiträge

\section{\) Auf eine fruchtbare Zusammenarbeit und gutes Gelingen!}

Die Gründung einer neuen Fachzeitschrift ist gerade heute mit Blick auf die üppige Medienlandschaft ein ambitioniertes Unterfangen und nicht denkbar ohne das große Engagement von Herausgebern, Gutachtern und Autoren. Wir danken allen "Machern" der Zeitschrift, insbesondere den Schriftleitern Herrn Professor Hube und Herrn Professor Petersen, und heißen sie herzlich willkom- 
men. Die Verlagsredaktion unterstützt Sie nach Kräften bei den anstehenden Aufgaben.

Liebe Leserinnen und Leser, wir freuen uns, wenn Das Knie Journal mit dem beschriebenen Konzept zu Ihrer unverzichtbaren Zeitschrift wird! Sie haben ausdrücklich die Möglichkeit, die Inhalte aktiv mitzugestalten, indem Sie einen Beitrag verfassen oder uns Ihr Feedback, Kritik und Anregungen zukommen lassen.

Bei der Lektüre der ersten Ausgabe von Knie Journal wünschen wir viel Vergnügen!

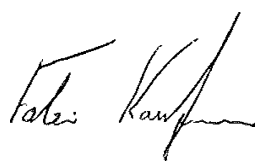

Fabian Kaufmann

Vorsitzender der Geschäftsführung

Springer Medizin Verlag GmbH

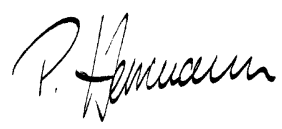

Dr. Paul Herrmann

Director Journals \& ePublishing

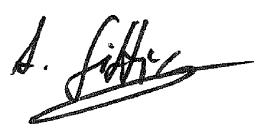

Anna Sittig

Managing Editor Knie Journal

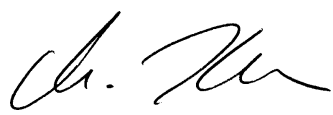

Monika Kretz

Head of Journals \& ePublishing 1

\section{Korrespondenzadresse}

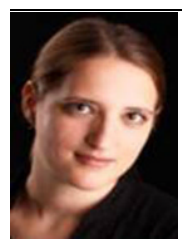

A. Sittig

Springer Medizin Verlag

$\mathrm{GmbH}$

Tiergartenstraße 17,

69121 Heidelberg,

Deutschland anna.sittig@springer.com

Interessenkonflikt. F. Kaufmann, P. Herrmann, A. Sittig und M. Kretz geben an, dass kein Interessenkonflikt besteht.

\section{Aktuelle Empfehlungen aus dem Springer-Verlag}

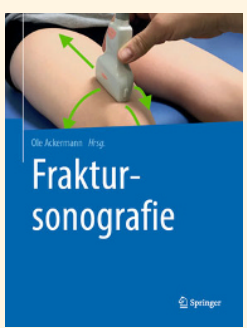

\section{Fraktursonografie}

O. Ackermann (Hrsg.)

1. Aufl. 2019, VIII, 128 S., 165 Abb., Hardvoer 69,99 €, ISBN 978-3-66258507-8

Vorgestellt werden die apparativen und fachlichen Voraussetzungen, die Indikationen und Abläufe und die möglichen Gefahren. Algorithmen, Pitfalls und Tipps zur rechtssicheren Dokumentation runden die Fraktursonografie in der Praxis ab.

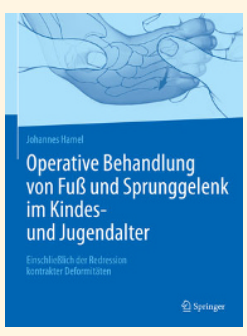

Operative Behandlung von Fuß und Sprunggelenk im Kindesund Jugendalter

J. Hamel

1. Aufl. 2019, X, 266 S., 872 Abb., Hardcover 74,99 €, ISBN 978-3-66256591-9

Exakte Beschreibung der vorgestellten operativen und redressierenden Verfahren. Didaktisch brillante Grafiken und Fotos illustrieren das praktische Vorgehen.

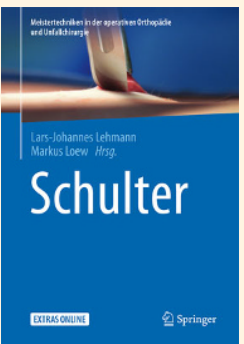

\section{Schulter}

L.-J. Lehmann, M. Loew (Hrsg.)

1. Aufl. 2018, XII, 293 S., 625 Abb., Hardcover 149,99€, ISBN 978-3-66254746-5

Die wesentlichen häufig vorkommenden, aber schwierig durchzuführenden Operationstechniken im Bereich "Schulter", dargestellt mit Step-by-step-Anleitungen und 230 brillanten Abbilungen.

\section{Ärztliche Begutachtung von A - Z}

E. Ludolph

1. Aufl. 2018, XX, 240 S., Hardcover $24,99 €$, ISBN 978-3-662-55866-9

Ärztliche

Begutachtung

von $A-Z$

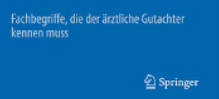

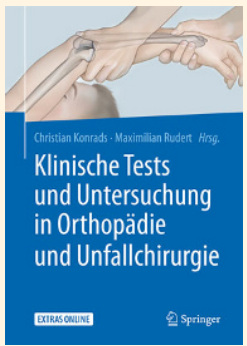

Damit Ärzte und Juristen die gleiche Sprache sprechen: Die wichtigsten Begriffe, die für die ärztliche Begutachtung bekannt sein müssen. Kompakte und vor allem präzise Erläuterungen. Sortiert von A - Z.

\section{Klinische Tests und Untersuchung in Orthopädie und} Unfallchirurgie

C. Konrads, M. Rudert (Hrsg.)

1. Aufl. 2018, XII, 184 S., 125 Abb., Softcover 24,99 €, ISBN 978-3-66255341-1

Das Buch enthält eine umfassende Darstellung aller relevanten klinischen Tests mit einer didaktisch präzisen Beschreibung des „How to do". Die praktische Umsetzung wird durch hervorragendes Bildmaterial veranschaulicht und Testergebnisse ausführlich interpretiert. 